\title{
Deformation of Borides in Nickel-based Superalloys: a Study of Segregation at Dislocations
}

\author{
Lola Lilensten ${ }^{1, *}$, Stoichko Antonov ${ }^{2,3}$, Dierk Raabe ${ }^{1}$, Sammy Tin $^{2}$, Baptiste Gault ${ }^{1,4}$, Paraskevas Kontis ${ }^{1}$ \\ ${ }^{1}$ Max-Planck-Institut für Eisenforschung, Max-Planck Strasse 1, 40237 Düsseldorf, Germany \\ ${ }^{2}$ Illinois Institute of Technology, 10 W. 32nd Street, Chicago, IL, 60616, USA \\ ${ }^{3}$ Beijing Advanced Innovation Center for Materials Genome Engineering, State Key Laboratory for \\ Advanced Metals and Materials, University of Science and Technology Beijing, Beijing 100083, China \\ ${ }^{4}$ Department of Materials, Imperial College, South Kensington, London SW7 2AZ, UK \\ * Corresponding author: 1.lilensten@ mpie.de
}

Boron is known to improve the ductility of polycrystalline superalloys at high temperatures by strengthening their grain boundaries. It is found at grain boundaries either in solid solution or in the form of borides [1,2]. However, we lack information on the behavior of borides during creep deformation. The present study investigates the segregation of solutes at dislocations in crept borides, aiming to understand the deformation of these grain boundary precipitates. Two polycrystalline nickel-based superalloys, namely RRHT3 and STAL15-CC, were crept, and segregation at dislocations within the borides was investigated by atom probe tomography (APT).

The RRHT3 alloy has a nominal composition 50Ni-9.9Al-18.3Co-13.3Cr-1.5Mo-4.6Nb-1.0Ta-0.8W$0.17 \mathrm{~B}$ (at.\%) and was deformed under creep at $750^{\circ} \mathrm{C}$ at $600 \mathrm{MPa}$ up to fracture. The STAL15-CC alloy with composition Ni-16.50Cr-5.50Co-0.60Mo-1.20W-10.00Al-2.40Ta-0.02Hf-0.5C-0.05B-0.01Zr (at.\%) was crept at $850^{\circ} \mathrm{C}$ at $235 \mathrm{MPa}$ up to fracture. Specimens for APT were prepared by site-specific lift-out from grain boundaries close to the fracture surface with a dual beam FEI Helios Nanolab 600 instrument. APT measurements were carried out on a Cameca LEAP ${ }^{\mathrm{TM}} 5000 \mathrm{XR}$ operated in laser pulsing mode at a pulse repetition rate of $125 \mathrm{kHz}$ and a pulse energy of $55 \mathrm{pJ}$, with a base temperature of $60 \mathrm{~K}$ and a detection rate of 15 ions every 1000 pulses.

Figure 1a shows an APT reconstruction from the RRHT3 alloy containing a $(\mathrm{Cr}, \mathrm{Mo})_{5} \mathrm{~B}_{3}$ boride neighboring a $\sigma$-precipitate. Linear features, which correspond to dislocations, are highlighted in the boride by a cobalt iso-composition surface (dark blue). A concentration profile across a dislocation denoted by arrow \#1 in Figure 1a is given in Figure 1b. The dislocation is enriched in cobalt and nickel that increase up to 19 at.\% and 7 at.\%, respectively and depleted in boron that drops to 15 at. $\%$ from approximately 36 at. $\%$. An enrichment in chromium is also measured, raising from 25 at. $\%$ to approximately 40 at.\%. Although cobalt and nickel are always found to segregate at these dislocations, this is less marked for chromium, whose composition fluctuates much more in the boride. Some of the dislocations seem to be in pairs, such as the ones in the red dashed frame in Figure 1a. A 2D composition profile map was extracted from this area for cobalt and nickel, see Figure 1c and 1d. The 2D concentration profile map of cobalt clearly shows two maxima next to each other, corresponding to the two dislocations. In contrast, nickel is found to segregate more in one dislocation than in the other one. This observation can be related to the different solute drag forces between the leading and trailing partial dislocations as found in other cases [3]. However, this observation needs to be further investigated by correlative transmission electron microscopy (TEM) and APT. 
Figure 1e shows an APT reconstruction from a deformed $\mathrm{Cr}_{5} \mathrm{~B}_{3}$ boride from the STAL15-CC alloy. Dislocations are highlighted by nickel isocomposition surfaces. Some dislocations were also observed to be in pairs, as in the case of RRHT 3, as shown in Figure 1e. A 1D composition profile across such a pair of dislocations is given in Figure 1f. Similar to RRHT3 alloy, nickel and cobalt were found to segregate at the dislocations. However, in this case there is no distinct depletion of boron and increase of chromium at the dislocations. It is believed that the different segregation behavior between the two

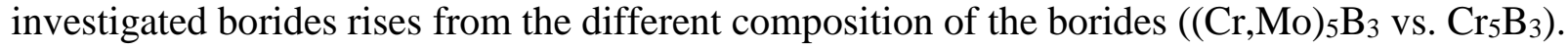

In summary, the present results show that nickel and cobalt segregate at dislocations in the borides, indicating that borides contribute to the deformation of superalloys and to the chemical alteration of the structure via solute drag at dislocations, as was already evidenced between the $\gamma$ matrix and $\gamma^{\prime}$ precipitates $[4,5]$. We believe that the level of segregation depends on the composition of the borides, which in this study is different for the borides between the RRHT3 and STAL15-CC alloy (see Figure 1). Finally, borides are often assumed to act as stress concentration sites facilitating crack initiation at grain boundaries during creep. However, our observations indicate that borides display plasticity at high temperature, suggesting that their deformation needs to be considered in understanding the mechanical performance of polycrystalline superalloys at elevated temperatures [6].

\section{References}

[1] D Tytko, P Choi, J Klöwer, A Kostka, G Inden, D Raabe, Acta Materialia 60 (2012), p. 1731.

[2] P Kontis, HAM Yusof, S Pedrazzini, M Danaie, KL Moore, PAJ Bagot, MP Moody, CRM

Grovenor, RC Reed, Acta Materialia 103 (2016), p. 688.

[3] S Hamadi, F Hamon, J Delautre, J Cormier, P Villechaise, S Utada, P Kontis, N Bozzolo, Metallurgical and Materials Transaction A 49 (2018), p. 4246.

[4] P Kontis, Z Li, DM Collins, J Cormier, D Raabe, B Gault, Scripta Materialia 145 (2018), p. 76.

[5] X Wu, SK Makineni, P Kontis, G Dehm, D Raabe, B Gault, G Eggeler, Materialia 4 (2018), p. 109.

[6] The authors thank Rolls Royce Corporation and Siemens Turbomachinery AB for providing the RRHT3 and STAL15-CC superalloys, respectively.
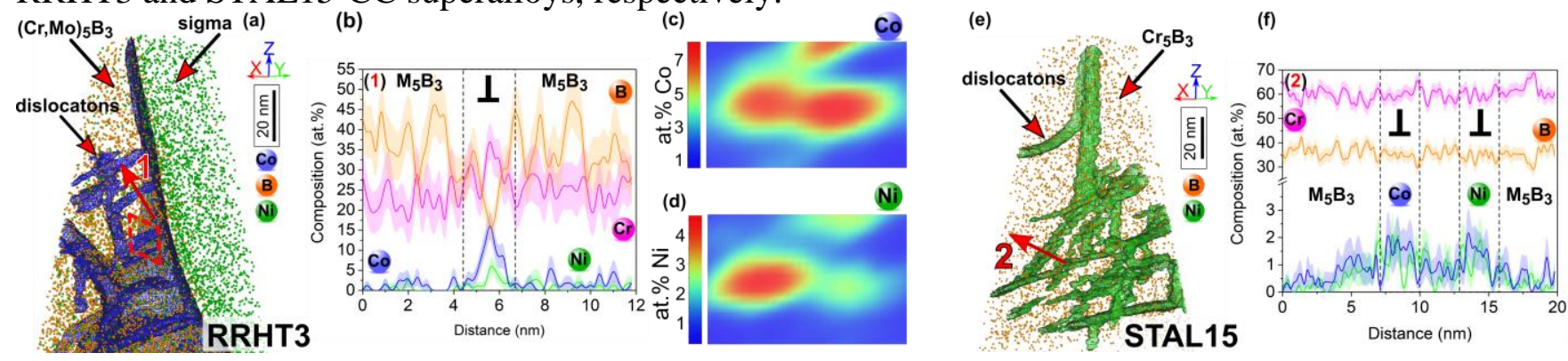

Figure 1. (a) APT reconstruction from the RRHT3 alloy showing a $(\mathrm{Cr}, \mathrm{Mo})_{5} \mathrm{~B}_{3}$ boride and a $\sigma$ precipitate. Dislocations are shown with an the iso-composition surface at 1 at.\% Co. (b) 1D concentration across a dislocation as denoted by arrow \#1 in (a). (c) $2 \mathrm{D}$ concentration profile map of cobalt corresponding to the area denoted by the red dashed box in (a). (d) 2D concentration profile map of nickel corresponding to the area denoted by the red dashed box in (a). (e) APT reconstruction of a $\mathrm{Cr}_{5} \mathrm{~B}_{3}$ boride from the STAL15-CC alloy, with dislocations shown with an iso-composition surface at 0.7 at.\% Ni. (f) $1 \mathrm{D}$ concentration profile across a pair of dislocations as denoted by arrow \#2 in (e). Error bars are shown as lines filled with color and correspond to the $2 \sigma$ counting error. 